\title{
Fatores sociodemográficos e emocionais associados à tolerância nas relações de amizade na pandemia pela covid-19
}

Sociodemographic and emotional factors associated with tolerance in friendship relationships in the covid-19 pandemic

Factores sociodemográficos y emocionales asociados con la tolerancia en las relaciones de amistad en la pandemia por covid-19

\section{Iel Marciano de Moraes FilhoI, Thais Vilela de Sousa ${ }^{\mathrm{II}}$, Francidalma Soares Sousa Carvalho Filha $^{\mathrm{III}}$, Mayara Cândida Pereira ${ }^{\mathrm{IV}}$, Jaiane de Melo Vilanovav ${ }^{\mathrm{V}}$, Rodrigo Marques da Silva ${ }^{\mathrm{VI}}$}

\begin{abstract}
Resumo: Objetivo: analisar a associação dos fatores sociodemográficos e emocionais ao nível de tolerância nas relações de amizade na pandemia pela covid-19. Método: trata-se de um estudo transversal realizado com 5291 indivíduos das cinco macrorregiões brasileiras de junho a julho de 2020. Aplicaram-se um questionário sociodemográfico, um questionário semiestruturado e o Instrumento de Avaliação da tolerância nas relações de amizade. Utilizou-se a regressão linear para análise de dados. Resultados: $88,9 \%$ consideram a tolerância nas relações de amizade satisfatória no domicílio, $82,8 \%$ tiveram ajuda de amigos para superar as tensões e $73 \%$ perceberam mudança nas relações de amizade durante a quarentena. Ser do gênero feminino, da raça branca e receber maior renda contribuíram para maiores níveis de tolerância nas relações de amizade. A maior escolaridade, receber ajuda de amigos durante a quarentena e a mudança nas relações de amizade levaram a menores níveis de tolerância. Conclusão: alguns fatores sociodemográficos e emocionais associam-se ao nível de tolerância nas relações de amizade, o que requer o desenvolvimento de ações estratégicas voltadas a tais fatores, especialmente aqueles modificáveis, como a renda e a escolaridade.
\end{abstract}

\footnotetext{
${ }^{\text {I }}$ Enfermeiro. Mestre em Ciências Ambientais e Saúde. Doutorando em Sociedade, Tecnologia e Meio Ambiente. Docente do curso de Enfermagem da Universidade Paulista (UNIP). Brasília, DF, Brasil. E-mail: ielfilho@yahoo.com.br ORCID ID: https://orcid.org/0000-0002-0798-3949

II Enfermeira. Mestre e Doutoranda em Enfermagem pelo Programa de Pós-Graduação em Enfermagem da Faculdade de Enfermagem da Universidade Federal de Goiás (UFG). Goiânia, Goiás, Brasil. E-mail: thais.fen@hotmail.com ORCID ID: https://orcid.org/0000-0002-7498-516X

III Enfermeira. Doutora em Saúde. Docente da Universidade Estadual do Maranhão (UEMA). Balsas, Maranhão, Brasil. E-mail: francidalmafilha@gmail.com ORCID ID: https://orcid.org/0000-0001-5197-4671

IVEnfermeira. Mestre e Doutoranda em Gerontologia da Universidade Católica de Brasília. Coordenadora do curso de Enfermagem da Universidade Paulista (UNIP). Brasília, DF, Brasil. E-mail: enfamayara@gmail.com ORCID ID: https://orcid.org/0000-0002-0242-6262

V Enfermeira. Especialista em Saúde da Família. Docente da Universidade Estadual do Maranhão (UEMA). Balsas, Maranhão, Brasil. E-mail: jai_vilanova@hotmail.com ORCID ID: https://orcid.org/0000-0001-8271-0177

VI Enfermeiro. Doutor em Enfermagem. Professor Titular da Faculdade de Ciências e Educação Sena Aires (FACESA). Valparaiso de Goiás, GO, Brasil. E-mail: marques-sm@hotmail.com ORCID ID: https://orcid.org/0000-0003-2881-9045
} 
Fatores sociodemográficos e emocionais associados a tolerância nas relações de... $\mid 2$

Descritores: Relações interpessoais; Amigos; Infecções por coronavírus; Pandemias; Isolamento social

\begin{abstract}
Objective: to analyze the association of sociodemographic and emotional factors with the level of tolerance in friendship relationships in the covid-19 pandemic. Method: this is a cross-sectional study conducted with 5,291 individuals from the five Brazilian macro-regions from June to July 2020. A sociodemographic questionnaire, a semi-structured questionnaire and the Instrument for Assessing tolerance in friendship relationships were applied. Linear regression was used for data analysis. Results: $88.9 \%$ considered tolerance in relationships of friendship satisfactory at home, $82.8 \%$ had help from friends to overcome tensions and $73 \%$ perceived a change in friendship relationships during quarantine. Being female, white and receiving higher income contributed to higher levels of tolerance in friendship relationships. Higher educational level, receiving help from friends during quarantine, and changing friendship relationships led to lower levels of tolerance. Conclusion: some sociodemographic and emotional factors are associated with the level of tolerance in friendship relationships, requiring the development of strategic actions aimed at such factors, especially those modifiable, such as income and schooling.
\end{abstract}

Descriptors: Interpersonal relations; Friends; Coronavirus infections; Pandemics; Social isolation

Resumen: Objetivo: analizar la asociación de factores sociodemográficos y emocionales con el nivel de tolerancia en las relaciones de amistad en la pandemia por covid-19. Método: se trata de un estudio transversal realizado con 5.291 individuos de las cinco macrorregiones brasileñas de junio a julio de 2020. Se aplicó un cuestionario sociodemográfico, un cuestionario semiestructurado y el Instrumento para evaluar la tolerancia en las relaciones de amistad. Se utilizó la regresión lineal para el análisis de datos. Resultados: el 88,9\% consideró que la tolerancia en las relaciones de amistad era satisfactoria en casa, el 82,8\% tenía ayuda de amigos para superar las tensiones y el 73\% percibió un cambio en las relaciones de amistad durante la cuarentena. Ser mujer, blanca y recibir mayores ingresos contribuyeron a mayores niveles de tolerancia en las relaciones de amistad. La educación superior, recibir ayuda de amigos durante la cuarentena y cambiar las relaciones de amistad llevaron a niveles más bajos de tolerancia. Conclusión: algunos factores sociodemográficos y emocionales están asociados con el nivel de tolerancia en las relaciones de amistad, lo que requiere el desarrollo de acciones estratégicas dirigidas a tales factores, especialmente aquellos modificables, como los ingresos y la escolarización.

Descriptores: Relaciones interpersonales; Amigos; Infecciones por coronavirus; Pandemias; Aislamiento social

\title{
Introdução
}

Desde o surgimento do novo coronavírus (SARS-CoV-2) na China em dezembro de 2019, a humanidade enfrentou uma grave crise global de saúde. Em países asiáticos como Tailândia, Japão, Coreia do Sul e Cingapura, novos casos se espalharam rapidamente para a Europa e outros continentes, o que levou a Organização Mundial da Saúde (OMS) a emitir um importante decreto de emergência em saúde pública. Ocorreu internacionalmente em 30 de janeiro de 2020 e estava em pandemia em 11 de março de 2020. ${ }^{1}$ De acordo com os dados disponíveis em 02 de 
3 | Moraes-Filho IM, Sousa TV, Carvalho-Filha FSS, Pereira MC, Vilanova JM, Silva RM

agosto deste ano, 213 países e regiões ao redor do mundo relataram 18.056,296 milhões de casos confirmados de covid-19, com um número de mortos que já passa dos 689.590 mil. ${ }^{2}$

No dia 03 de fevereiro, o Ministério da Saúde do Brasil declarou estado de Emergência em Saúde Pública de Importância Nacional (ESPIN) em decorrência da infecção humana pelo novo coronavírus, isso ocorreu trinta e três dias após a notificação inicial da China, e quatro dias após OMS declarar a covid-19 uma emergência internacional. ${ }^{3}$

No Brasil, conflitos entre instituições técnicas e políticas têm afetado na resposta federal à pandemia. As narrativas dos discursos do presidente exacerbaram ainda mais a tensão entre a implementação de medidas de isolamento social horizontais e o funcionamento da economia, que logo privilegia a economia em detrimento das medidas de prevenção à doença. ${ }^{4}$

Ademais, as pandemias estão associadas a uma série de estressores sociais e clínicos que criam perturbações graves em vários níveis. As pandemias estão relacionadas à confusão, medos, incertezas e às prováveis mortes de amigos e entes queridos. Ainda, nessas situações, estão presentes uma variedade de estressores sociais, como: perda econômica e de emprego; isolamento social; colapso dos sistemas de saúde; bem como mudanças drásticas no modo e no estilo de vida, incluindo os horários das refeições, a qualidade e tempo de sono. Nesse sentido, observou-se um aumento da morte em decorrência do coronavírus seguido por abalos psicológicos, como estresse, medo e ansiedade na população. ${ }^{5}$

Assim, a abordagem de resposta à situação pandêmica da covid-19 deve passar pelo esclarecimento não só do número de casos e da transmissibilidade, mas também pelo impacto da epidemia no que diz respeito às relações humanas. Por meio de pesquisas científicas, é preciso identificar quais são os fatores associados à infecção, gravidade ou morte, quais os grupos de maior probabilidade de evolução desfavorável e se é possível concentrar esforços na prevenção desse desfecho clínico negativo e/ou morte e logo, dos possíveis impactos sociais. ${ }^{6}$ 
Fatores sociodemográficos e emocionais associados a tolerância nas relações de... $\mid 4$

$\mathrm{Na}$ ausência de relacionamentos interpessoais importantes, a ansiedade e a depressão podem aparecer ou piorar. Da mesma forma, a quarentena se torna difícil para muitas pessoas receberem intervenções e aditivos psicoterapêuticos. Essas descobertas destacam a importância das conexões sociais na minimização do impacto negativo do isolamento da covid-19. ${ }^{5}$

Em se tratando de construto, as amizades podem ser estabelecidas a partir de quatro fatores. O primeiro é o Ambiental que está relacionado com a proximidade física e residual entre as pessoas. Depois, tem-se o fator Situacional, conceituado como a relação entre interação e sequência. O fator Individual inclui normas de inclusão e exclusão que são praticadas na préseleção de candidatos a amigos. Por fim, o fator Diádico envolve o apreço recíproco e mútuo de julgamentos iniciais que um indivíduo faz do outro. Nota-se, então, que a amizade envolve fatores como idade, sexo, religião, ideologias, escolaridade, etnias e raça. ${ }^{7}$ Tendo em vista o atual isolamento das pessoas como medidas de prevenção de contágio da covid-19, as relações sociais podem se encontrar prejudicadas, com impacto direto ao nível de tolerâncias nas relações de amizade. ${ }^{8}$

Nesse contexto, questiona-se: Os fatores sociodemográficos e emocionais associam-se ao nível de tolerância nas relações de amizade durante a pandemia pela covid-19? Por conseguinte, o objetivo deste estudo foi analisar a associação dos fatores sociodemográficos e emocionais ao nível de tolerância nas relações de amizade na pandemia pela covid-19.

\section{Método}

Trata-se de um estudo transversal, realizado com a população brasileira das cinco macrorregiões (Norte, Nordeste, Sudeste, Sul e Centro-Oeste) entre junho e julho de 2020. Foram incluídos indivíduos com mais de 18 anos, com acesso à internet por meio de inscrição às plataformas digitais sociais de relacionamento ou mensagens. Foram excluídos os participantes que não preencheram de forma totalitária as questões do instrumento de pesquisa. Para tanto, utilizou-se uma amostra não probabilística do tipo conveniência, o que acarretou encerramento 
5 | Moraes-Filho IM, Sousa TV, Carvalho-Filha FSS, Pereira MC, Vilanova JM, Silva RM

da coleta de dados quando obtido um número mínimo de 5.000 pessoas, incluindo todas as macrorregiões brasileiras.

Os dados foram coletados por meio dos seguintes instrumentos autoaplicáveis: questionário sociodemográfico; questionário semiestruturado sobre o padrão das relações interpessoais de cada participante a respeito da pandemia de covid-19; e Instrumento de Avaliação da tolerância nas relações de amizade (ATRA). ${ }^{9}$ Esses foram digitados no formulário do Google ${ }^{\circledR}$ e submetidos pelas plataformas sociais: Facebook ${ }^{\circledR}$, Twitter $^{\circledR}$, Whatsapp ${ }^{\circledR}$ e Instragram ${ }^{\circledR}$. Tais instrumentos só puderam ser respondidos após a confirmação digital de aceite em participar do estudo mediante assinatura do Termo de Consentimento Livre e Esclarecido (TCLE).

O questionário sociodemográfico, construído pelos autores, envolveu as seguintes variáveis: data de nascimento, gênero, orientação sexual, raça, escolaridade, instituição de ensino superior, renda mensal, região de moradia e com quem reside (sozinho (a) ou não). O questionário semiestruturado abordou as emoções a respeito da pandemia de covid-19 e conteve as seguintes perguntas: No seu ambiente familiar, a tolerância de amizade é satisfatória? Os seus amigos lhe ajudaram a superar as tensões vivenciadas durante o período de quarentena/isolamento social da covid-19? Você acredita que houve mudanças nas suas relações de amizade desde o início da quarentena/isolamento social em virtude da pandemia de covid-19?

A ATRA $^{9}$ foi construída no ano de 2019 para avaliação da tolerância nas relações de amizade. Sua estruturação foi baseada na Análise semântica de evidências e fundamentada no processo de construção de escalas psicométricas. ${ }^{10-11}$ Ela é composta por 21 itens dispostos em escala tipo likert de cinco pontos, em que: 1 - concordo totalmente, 2 - concordo parcialmente, 3 - não concordo e nem discordo, 4 - discordo parcialmente e 5 -discordo totalmente. Por se tratar de escala inversa, é necessário a inversão da mesma em todos os itens antes da realização da análise dos dados. A partir da soma das pontuações assinaladas em cada item, obtêm-se os escores do grau da tolerância de amizade, sendo que quanto menor a pontuação, maior a 
Fatores sociodemográficos e emocionais associados a tolerância nas relações de... 6

tolerância das relações de amizade. Com base na média geral para a população pesquisada, a tolerância de amizade é dicotomizada em alta tolerância (quando o indivíduo apresenta escore de tolerância em relação a amizade superior à média da população) e baixa tolerância (quando o indivíduo apresenta escore de tolerância quanto à amizade abaixo da média da população). Os itens de maior média representam as situações em que há maior tolerância nas relações de amizade. ${ }^{9}$ Sendo assim, o desfecho analisado foi o nível de tolerância nas relações de amizade.

Para organização e análise dos dados, foi construído um banco de dados no programa Excel (Office 2019) e utilizado o programa Statistical Package for Social Science (SPSS), versão 25.0. As variáveis qualitativas foram apresentadas em valores absolutos (n) e percentuais (\%). As variáveis quantitativas foram expostas em medidas descritivas: valores mínimos e máximos, média e desvio padrão. A normalidade dos dados foi avaliada por meio do teste KolmogorovSmirnov. Para a análise, utilizou-se a regressão linear simples, com método backward para a seleção das variáveis, o $\mathrm{R}^{2}$ Ajustado como indicador de ajuste do modelo e o ANOVA (Teste F) como indicador de significância dele. A correlação parcial e o respectivo valor de $\mathrm{p}$ foram utilizados como critérios de exclusão de variáveis nos modelos testados. Em cada modelo, variáveis com a menor correlação parcial foram excluídas até a obtenção do modelo final. O efeito de cada preditor sobre o desfecho nível de tolerância nas relações de amizade foi avaliado por meio dos valores de Beta, com significância estatística de 5\%. Foram avaliados os pressupostos de linearidade das relações e normalidade dos erros para definição do modelo final. A possível existência de multicolinearidade foi avaliada em cada variável dos modelos por meio do Fator de Inflação da Variância (VIF), sendo aceitos valores de 1 a 10 como adequados. ${ }^{12}$

Este projeto faz parte de um estudo maior intitulado: Tolerância nas relações de amizade no contexto da pandemia de COVID- 19. O projeto foi submetido, via plataforma Brasil, para apreciação no Comitê de Ética em Pesquisa (CEP), sendo aprovado sob parecer número 4.113.127 em 26 de junho de 2020. Além disso, atendendo às Diretrizes e Normas 
7 | Moraes-Filho IM, Sousa TV, Carvalho-Filha FSS, Pereira MC, Vilanova JM, Silva RM

Regulamentadoras de Pesquisas Envolvendo Seres Humanos (Resolução CNS 466/12), o Termo de Consentimento Livre e Esclarecido foi apresentado e assinado (em modo online) pelos que aceitaram participar da pesquisa antes da resposta aos instrumentos.

\section{Resultados}

Das 5315 pessoas que foram convidadas para o estudo, 24 não aceitaram participar, o que levou a uma população de acesso de 5291 participantes. A análise do Alfa de Cronbach demonstrou valor de 0,80 para os 21 itens do ATRA o que atesta confiabilidade satisfatória ao instrumento. Na Tabela 1, apresentam-se os dados de caracterização sociodemográfica da população pesquisada.

Tabela 1- Caracterização sociodemográfica da população pesquisada (n=5291). Brasil. 2020.

\begin{tabular}{llrr}
\hline \multicolumn{1}{c}{ Variáveis } & \multicolumn{1}{c}{ Categorias } & $\begin{array}{r}\text { Frequência } \\
\text { Absoluta } \\
\text { (n) }\end{array}$ & $\begin{array}{r}\text { Frequência } \\
\text { Relativa (\%) }\end{array}$ \\
\hline Faixa etária & 18 a 29 anos & 3403 & 64,3 \\
& 30 a 49 anos & 1450 & 27,4 \\
& 50 a 59 anos & 196 & 3,7 \\
Total & Z60 anos & 242 & 4,6 \\
Gênero & & 5291 & 100,0 \\
& Feminino & 4310 & 81,5 \\
Total & Masculino & 956 & 18,1 \\
Orientação Sexual & Outro & 25 & 0,4 \\
& & 5291 & 100,0 \\
& Heterossexual & 3768 & 71,2 \\
& Homossexual & 621 & 11,7 \\
Total & Bissexual & 786 & 14,9 \\
Instituição de Ensino & Outros & 89 & 1,7 \\
Superior & Não responderam & 27 & 0,5 \\
& & 5291 & 100,0 \\
& Pública & 1743 & 32,9 \\
Total & Privada & 2135 & 40,4 \\
Escolaridade & Nenhuma instituição de & 1413 & 26,7 \\
& ensino no momento & 5291 & 100,0 \\
& & 55 & 1,0
\end{tabular}


Fatores sociodemográficos e emocionais associados a tolerância nas relações de... $\mid 8$

\begin{tabular}{|c|c|c|c|}
\hline & Ensino Médio & 1212 & 22,9 \\
\hline & Ensino Superior & 2717 & 51,4 \\
\hline & Pós-graduação & 946 & 17,9 \\
\hline & Mestrado & 265 & 5,0 \\
\hline & Doutorado & 96 & 1,8 \\
\hline Total & & 5291 & 100,0 \\
\hline \multirow[t]{2}{*}{ Mora Sozinha(o) } & Sim & 693 & 13,1 \\
\hline & Não & 4598 & 86,9 \\
\hline Total & & 5291 & 100,0 \\
\hline \multirow[t]{6}{*}{ Raça } & Amarela & 149 & 2,8 \\
\hline & Branca & 2685 & 50,7 \\
\hline & Indígena & 20 & 0,4 \\
\hline & Parda & 1777 & 33,6 \\
\hline & Preta & 615 & 11,6 \\
\hline & Outra & 45 & 0,9 \\
\hline Total & & 5291 & 100,0 \\
\hline \multirow[t]{5}{*}{ Renda Mensal } & 20 ou mais salários mínimos & 204 & 3,9 \\
\hline & $\begin{array}{l}\text { Entre } 10 \text { e } 20 \text { salários } \\
\text { mínimos }\end{array}$ & 632 & 11,9 \\
\hline & Entre 4 e 10 salários mínimos & 1647 & 31,1 \\
\hline & Entre 2 e 4 salários mínimos & 1549 & 29,3 \\
\hline & Até 2 salários mínimos & 1259 & 23,8 \\
\hline Total & & 5291 & 100,0 \\
\hline \multirow[t]{6}{*}{ Região de moradia } & Centro-Oeste & 1789 & 33,8 \\
\hline & Sul & 528 & 10,0 \\
\hline & Sudeste & 1760 & 33,3 \\
\hline & Nordeste & 984 & 18,6 \\
\hline & Norte & 182 & 3,4 \\
\hline & Fora do Brasil & 48 & 0,9 \\
\hline Total & & 5291 & 100,0 \\
\hline
\end{tabular}

Observa-se o predomínio de participantes com idades entre 18 e 29 anos (64,3\%), do sexo feminino $(81,5 \%)$, heterossexuais $(71,2 \%)$, da raça/cor branca $(50,7 \%)$, que recebem entre 4 e 10 salários mínimos (31,1\%). Eles estão vinculados a instituições privadas de ensino $(40,4 \%)$, possuem ensino superior completo $(51,4 \%)$ e são oriundos do Centro-Oeste $(33,8 \%)$ e Sudeste (33,3\%). Na Tabela 2, apresenta-se a avaliação do padrão das relações interpessoais e as emoções de cada participante a respeito da pandemia de covid-19. 
9 | Moraes-Filho IM, Sousa TV, Carvalho-Filha FSS, Pereira MC, Vilanova JM, Silva RM

Tabela 2- Padrão das relações interpessoais e emoções de cada participante a respeito da pandemia de covid-19, Brasil. ( $\mathrm{n}=5291)$.

\begin{tabular}{lrrr}
\hline Descrição & & $\begin{array}{r}\text { Frequência } \\
\text { Absoluta } \\
\text { (n) }\end{array}$ & $\begin{array}{r}\text { Frequência } \\
\text { Relativa } \\
(\%)\end{array}$ \\
\hline No seu ambiente familiar, a tolerância de amizade é satisfatória? & Sim & 4704 & 88,9 \\
& Não & 587 & 11,1 \\
& Sim & 4382 & 82,8 \\
Os seus amigos lhe ajudaram a superar as tensões vivenciadas & Não & 909 & 17,2 \\
durante o período de quarentena/isolamento social da covid-19? & Sim & 3863 & 73,0 \\
Você acredita que houve mudanças nas suas relações de amizade & Não & 1428 & 27,0 \\
desde o início da quarentena/isolamento social em virtude da & & & \\
pandemia de covid-19? & &
\end{tabular}

Observa-se predomínio de indivíduos que consideram satisfatória a tolerância de amizade no ambiente domiciliar (88,9\%); cujos amigos ajudaram a superar as tensões vivenciadas durante o período de quarentena/isolamento social $(82,8 \%)$; e que acreditam ter havido mudança nas relações de amizade desde o início da quarentena (73\%). Na Tabela 3, apresentam-se os indicadores de ajuste e processo de exclusão de variáveis entre os modelos inicial e final testados.

Tabela 3- Indicadores de Ajuste e processo de exclusão de variáveis entre os modelos inicial e final testados ( $\mathrm{n}=5291)$. Brasil, 2020.

\begin{tabular}{lcccc}
\hline $\begin{array}{l}\text { Modelos } \\
\text { testados }\end{array}$ & $\mathbf{R}^{2}$ Ajustado & $\begin{array}{c}\text { Variável excluída a cada } \\
\text { modelo }\end{array}$ & $\begin{array}{c}\text { Correlação } \\
\text { parcial }^{*}\end{array}$ & $\begin{array}{c}\text { ANOVA } \\
\text { (Teste F) para } \\
\text { o Modelo }\end{array}$ \\
\hline Modelo Inicial & 0,290 & - & - & $\mathrm{p}<0,001$ \\
Modelo 2 & 0,290 & Orientação Sexual & 0,006 & $\mathrm{p}<0,001$ \\
Modelo 3 & 0,290 & Morar sozinho(a) & $-0,011$ & $\mathrm{p}<0,001$ \\
Modelo 4 & 0,290 & Instituição de Ensino & $-0,017$ & $\mathrm{p}<0,001$ \\
Modelo Final & 0,280 & Região de Moradia & 0,018 & $\mathrm{p}<0,001$ \\
\hline
\end{tabular}

*Parâmetro de exclusão da variável.

No modelo de regressão inicialmente obtido, o gênero, a raça, a escolaridade, a renda mensal, receber ajuda de amigos na quarentena e mudança nas relações de amizade durante a quarentena foram as variáveis que contribuíram significativamente para a tolerância nas 
relações de amizade. Todavia, devido a valores de p superiores a 0,05 e correlação parcial baixa, algumas variáveis foram excluídas até a obtenção do modelo final: orientação sexual, morar sozinha(o), instituição de ensino superior e região de moradia. Os valores de VIF variaram de 1,004 a 1,147 para as variáveis preditoras inseridas no modelo final, o que implica inexistência de multicolinearidade das variáveis na explicação do desfecho. Tal modelo foi estatisticamente significativo $(\mathrm{p}<0,05)$ e o conjunto de preditores explicou $40 \%$ do desfecho (Tabela 4$)$.

Tabela 4-Modelo final de regressão linear dos fatores sociodemográficos e emocionais sobre o nível de tolerância nas relações de amizade durante a pandemia pela covid-19 ( $\mathrm{n}=5291)$. Brasil, 2020.

\begin{tabular}{lccc}
\hline Variáveis preditoras & $\boldsymbol{\beta}$ & P valor & $\begin{array}{c}\text { Fator de } \\
\text { Inflação da } \\
\text { variância } \\
\text { (VIF) }\end{array}$ \\
\hline (Constant) & & & - \\
Gênero (Feminino) & 3,473 & 0,000 & 1,005 \\
Raça (Branca) & 0,100 & $<0,001^{*}$ & 1,048 \\
Escolaridade & 0,018 & $0,009^{*}$ & 1,103 \\
Renda mensal & $-0,053$ & $<0,001^{*}$ & 1,147 \\
Receber ajuda de amigos durante a quarentena (Sim) & 0,033 & $<0,001^{*}$ & 1,013 \\
Mudança nas relações de amizade durante a quarentena (Sim) & $-0,079$ & $<0,001^{*}$ & 1,004 \\
\hline
\end{tabular}

${ }^{*}$ Valor estatisticamente significativo $(\mathrm{p}<0,05)$.

$\mathrm{Na}$ Tabela 4, de um lado, verifica-se que ser do gênero feminino, da raça branca e receber maior renda foram fatores que contribuíram significativamente para maiores níveis de tolerância nas relações de amizade. Por outro lado, maior escolaridade, receber ajuda de amigos durante a quarentena e a mudança nas relações de amizade durante a pandemia levaram a menores níveis de tolerância nas relações de amizade.

\section{Discussão}


11 | Moraes-Filho IM, Sousa TV, Carvalho-Filha FSS, Pereira MC, Vilanova JM, Silva RM

Em estudo análogo ${ }^{8}$ também realizado durante a pandemia/isolamento social, das 16.440 respostas válidas da amostra, 69\% das pessoas eram do sexo feminino, corroborando com estes achados. Em relação às faixas de renda, observou-se que 34\% das pessoas declararam receber até 2 salários mínimos em oposição a este estudo em que 31,1\% recebem entre 4 e 10 salários mínimos. ${ }^{8}$ Isso pode ser explicado pelo fato de os participantes serem predominantemente das regiões central e sudeste do país, desta forma não representando a realidade brasileira em que a renda média mensal no $1^{\circ}$ trimetre de 2020 ficou em torno de $R \$ 2.398,00$ como dados da Pesquisa Nacional por Amostra de Domicílios Contínua. ${ }^{13}$

No censo da educação superior realizado pelo Instituto Nacional de Estudos e Pesquisas Educacionais Anísio Teixeira no ano de 2017, percebe-se que a maioria dos indivíduos estão vinculadas a instituição privadas de ensino, ${ }^{14}$ corroborando com estes achados que ficou em torno de 40,4\% .Neste estudo, houve também predomínio de mulheres da raça branca, achado que vai ao encontro de outra investigação realizada em 2015, a qual demostrou que o grupo das mulheres brancas é o mais bem distribuído dentre os trabalhadores com ensino superior e tem maiores rendas financeiras. ${ }^{15}$

Uma pesquisa realizada em duas universidades privadas uma em Bangkok e a outra na Tailândia com 438 funcionários durante a pandemia de covid-19, sugere que o apoio social proteja as pessoas dos efeitos nocivos dos eventos estressantes, influenciando no que eles pensam e como eles lidam com os eventos. Dessa forma, o suporte social refere-se a uma provisão da rede de recursos psicológicos e materiais destinados a beneficiar a capacidade de um indivíduo de lidar com o estresse. ${ }^{16}$ Neste estudo, indivíduos que consideram satisfatória sua tolerância de amizade no ambiente domiciliar e cujos amigos ajudaram a superar as tensões vivenciadas durante o período de quarentena/isolamento social demonstram que o apoio social é extremamente benéfico para o bem-estar e saúde mental em relacionamentos. ${ }^{17}$ 
Outro estudo realizado em 28 instituições de ensino superior da Ucrânia e da Polônia com 690 estudantes também ressaltou que a proteção contra possíveis efeitos de estresse nas situações de crise, se baseia em treinamentos, comunicação, conversa com amigos nas redes sociais. Estes fatores ajudam a enfrentar os riscos de adoecimento psicológico durante as medidas de isolamento social. ${ }^{18}$

Verifica-se que gênero feminino, raça branca e renda mensal possuem efeito significativo e direto ao nível de tolerância nas relações de amizade. Isto pode ser explicado pelo fato de que a amostra se compõe por adultos jovens, na faixa etária entre 18 e 29 anos e que reconhecem, como fatores preditores para uma boa amizade, os seguintes aspectos: a similaridade em termos de instrução, classe social, background familiar ou estágio do ciclo familiar. Logo, eles se sustentam na abertura mútua e na fraqueza pessoal, o que pode justificar as adversidades vivenciadas durante a pandemia/isolamento social e a contribuição significativa dos fatores de orientação sexual, morar sozinha(o), instituição de ensino superior e região de moradia para a tolerância nas relações de amizade. ${ }^{19-20}$

Ademais, pessoas com maior escolaridade apresentam menores níveis de tolerância nas relações de amizade. Isso se opõem a outros estudos, nos quais se verifica que a vivência universitária exerce impacto sobre aspectos sociais, técnicos, cognitivos e afetivos dos estudantes, tornando-os adultos que apresentam melhores desempenhos empáticos em relacionamentos diversos. ${ }^{21-22}$

Outro fator destacado foi de receber ajuda de amigos durante a quarentena nos menores níveis de tolerância. Muitas vezes este fator ocorre devido aos familiares e amigos não saberem como dispensar esta ajuda. Por isso, é importante dar a eles a chance de fazer isso e dizer quais as necessidades reais para ser ajudado. ${ }^{23} \mathrm{E}$ de encontro com tais achados, um estudo realizado na China com 15 jovens, demonstrou que eles especialmente quando são altamente instruídos demonstram não precisarem de apoio psicológico profissional. ${ }^{17}$ As mudanças nas relações de amizade durante a 
pandemia podem ser explicadas devido ao estreitamento social ocasionados por conta do carinho, da preocupação, da fraternidade, do humor, da saudade, da amizade; ou, pela solidariedade de todas as pessoas que passam pelo o mesmo mal, ocasionado pelo medo do contágio. ${ }^{2425}$

Como limitações do estudo, destacam-se o fato de não retratar equitativamente todos os extratos sociais e etários da população brasileira segundo as macrorregiões, o que permitiria possíveis comparações das variáveis e desfecho entre as diferentes regiões brasileiras. Além disso, não há muitos estudos com esta abordagem na literatura científica, o que limitou a ampla discussão e debate sobre o tema. Nesse sentido, sugere-se a realização de outros estudos nacionais, com amostragem proporcional estratificada, a fim de que possa analisar e comparar as especificidades das macrorregiões durante a pandemia.

\section{Conclusão}

Observa-se o predomínio de participantes com idades entre 18 e 29 anos, do sexo feminino, heterossexuais, da raça/cor branca, que recebem entre 4 e 10 salários mínimos. Eles estão vinculados a instituições privadas de ensino, possuem ensino superior completo e são oriundos do Centro-Oeste e Sudeste.

Os indivíduos consideraram satisfatória a tolerância de amizade no ambiente domiciliar; os amigos ajudaram a superar as tensões vivenciadas durante o período de isolamento social; e eles acreditam ter havido mudança nas relações de amizade desde o início da quarentena. Ademais, ser do gênero feminino, da raça branca e receber maior renda foram fatores que contribuíram significativamente para maiores níveis de tolerância nas relações de amizade. Já a maior escolaridade, receber ajuda de amigos durante a quarentena e a mudança nas relações de amizade durante a pandemia levaram a menores níveis de tolerância nas relações de amizade.

A identificação dos fatores associados ao nível de tolerância nas relações de amizade durante a pandemia permitirá o desenvolvimento de ações estratégicas voltadas a tais fatores, 
Fatores sociodemográficos e emocionais associados a tolerância nas relações de... | 14

especialmente aqueles modificáveis, como a renda e a escolaridade. Com o fechamento de diversos serviços, as dificuldades econômicas tornaram-se mais intensas e o isolamento modificou as relações humanas que se tornaram muito dependentes da tecnologia. Frente a isso, é preciso que gestores e profissionais de saúde adotem políticas e programas que considerem os aspectos econômicos e as novas formas de se relacionar como elementos que impactam na saúde física e mental da população.

Assim, mudando a perspectiva do cuidado, melhorando as possibilidades individuais e coletivas de assistência, pautando nas novas adversidades vivenciadas com vistas a manter uma saúde mental favorável da população, nota-se a intenção de preservar sentimentos de estima e perseverança que ajudaram no enfrentamento das adversidades ocasionadas pela pandemia.

\section{Referências}

1. Aquino EML, Silveira IH, Pescarini JM, Aquino R, Souza- Filho JA, Rocha AS, et al. Medidas de distanciamento social no controle da pandemia de COVID-19: potenciais impactos e desafios no Brasil. Ciênc Saúde Colet. 2020; 25(Suppl1): 2423-46. Doi: 10.1590/1413-81232020256.1.10502020.

2. Worldometer. Countries where COVID-19 has spread [Internet]. 2020 [acesso em 2020 jul 28]. Disponível em: https://www.worldometers.info/coronavirus/countries-where-coronavirus-has-spread/

3. Brasil. Portaria no 188, de 3.2.2020, publicada no DOU de 4.2.2020[Internet]. 2020 [acesso em 2020 ago 2]. Disponível em: http://bvsms.saude.gov.br/bvs/saudelegis/gm/2020/prt0188_04_02_2020.html

4. Tavares AB, Silveira F, Paes-Sousa R. Proteção Social e COVID-19: a resposta do Brasil e das maiores economias da América Latina. Rev NAU Social [Internet]. 2020 [acesso 2020 jun 01]; 11(20): 111-29. Disponível em:https://portalseer.ufba.br/index.php/nausocial/article/view/36599/21017

5. Martínez-Taboas, A. Pandemias, COVID-19 y Salud Mental: ¿Qué Sabemos Actualmente? Rev Cari Psicol. 2020; 4(2), 143-52. Doi: 10.37226/rcp.v4i2.4907

6. Lipsitch M, Swerdlow DL, Finelli L. Defining the epidemiology of Covid-19 - studies needed. N Engl J Med. 2020; 382(13):1194-95. Doi: 10.1056/NEJMp2002125

7. Santos AS, Oliveira CT, Dias ACG. Características das relações dos universitários e seus pares: implicações na adaptação acadêmica. Psicol teor prat[Internet]. 2015 [acesso 2020 jun 10]; 17(1):150-63. Disponível em: http://pepsic.bvsalud.org/scielo.php?script=sci_arttext\&pid=S1516-36872015000100013. 
8. Bezerra ACV, Silva CEM, Soares FRG, Silva JAM. Fatores associados ao comportamento da população durante o isolamento social na pandemia de COVID-19. Ciênc. Saúde Colet. 2020; 25(Suppl1):24112421. Doi: $10.1590 / 1413-81232020256.1 .10792020$

9. Moraes-Filho IM, Carvalho LF, Melo LE, Marcelo MRD, Santos YM, Faria MRGV. Construção do instrumento para avaliação da tolerância nas relações de amizade. Rev Cient Sena Aires[Internet]. 2019[acesso 2020 jun 10]; 8(1): 71-9. Disponível em: http://revistafacesa.senaaires.com.br/index.php/revisa/article/view/383.

10. França AB, Schelini PW. Análise semântica e evidências de validade da escala metacognitiva para idosos. Aval psicol [Internet]. 2014[acesso 2020 jun 10]; 13(3): 333-41 Disponível em: http://pepsic.bvsalud.org/pdf/avp/v13n3/v13n3a05.pdf.

11. Reppold CT, Gurgel LG, Hutz CS. O processo de construção de escalas psicométricas. Aval psicol[Internet]. 2014[acesso 2020 jun 10];13(2):307-310. Disponível em: http://pepsic.bvsalud.org/pdf/avp/v13n2/v13n2a18.pdf.

12.Favero LP, Belfiore P. Manual de Análise de Dados - Estatística e Modelagem Multivariada com Excel $^{\circledR}$, SPSS $^{\circledast}$ e Stata $^{\circledR} .1^{\text {a }}$. ed. São Paulo: GEN LTC; 2017.

13.Instituto Brasileiro de Geografia e Estatística. Pesquisa Nacional por Amostra de Domicílios Contínua 1o trimestre [Internet]. 2020 [acesso em 2020 jul 28]. Disponível em: https://www.ibge.gov.br/indicadores.html.

14.Instituto Nacional de Estudos e Pesquisas Educacionais Anísio Teixeira. Censo da Educação Superior [Internet]. 2017 [acesso em 2020 jul 28]. Disponível em: http://portal.mec.gov.br/docman/setembro-2018pdf/97041-apresentac-a-o-censo-superior-u-ltimo/file.

15. Souza SL, Siqueira LN. O impacto da segregação ocupacional por gênero e raça na desigualdade de renda no Brasil em três décadas (1986-2015). Rev Latinoam Poblac. 2020; 14(27):41-76. Doi: 10.31406/relap2020.v14.i12.n27.2

16. Charoensukmongkol P, Phungsoonthorn T. The effectiveness of supervisor support in lessening perceived uncertainties and emotional exhaustion of university employees duringthe COVID-19 crisis: the constraining role of organizational intransigence. J Gen Psychol. 2020. Doi: 10.1080/00221309.2020.1795613

17. Carter E, Moname R, Peccoralo L, Chipman J, Chacko S, Duran A, et al. Missed opportunities to engage patients in collaborative care challenge program sustainability: A qualitative study. Gen hosp psychiatr. 2020; 66:81-88. Doi: 10.1016/j.genhosppsych.2020.07.006

18.Lobanova A, Zoska Y, Długosz P. Intentions of Ukrainian and Polish students in quarantine measures during the Covid-19 pandemic: a sociological dimension. EPST. 2020;3(1):104-17. Doi: 10.15421/342012 
Fatores sociodemográficos e emocionais associados a tolerância nas relações de... | 16

19.Senna SRCM, Dessen MA. Reflexões sobre a saúde do adolescente brasileiro. Psicol saúde doenças. 2015; 16(2): 217-29. Doi: 10.15309/15psd160208

20. Sousa DA, Santos CE. Redes sociais e relacionamentos de amizade ao longo do ciclo vital. Rev psicopedag [Internet]. 2011 [acesso 2020 jun 10]; 28(85): 53-66. Disponível em: http://pepsic.bvsalud.org/pdf/psicoped/v28n85/06.pdf

21.Pitta KB, Santos LAD, Escher CA, Bariani ICD. Estilos cognitivos de estudantes de psicologia: impacto da experiência em iniciação científica. Psicol Esc Educ [Internet]. 2000 [acesso 2020 jun 10];(4) 2: 41-9. Disponível em: https://www.scielo.br/pdf/pee/v4n2/v4n2a05.pdf

22.Pinho VD, Fernandes CS, Falcone EMO. A influência da idade e da escolaridade sobre a experiência empática de adultos. Estud pesqui [Internet]. 2011[acesso 2020 jun 10];11(2):456-71. Disponível em: http://pepsic.bvsalud.org/pdf/epp/v11n2/v11n2a06.pdf

23. Associação Brasileira de Estudos Cemiteriais. Rede de Apoio às Famílias com Covid. Segura a Onda. Guia para pessoas que perdem um ente querido em tempos de coronavírus (COVID-19). Santa Catarina: Associação Brasileira de Estudos Cemiteriais; 2020. Disponível em: https://seguraaonda.com.br/wpcontent/uploads/2020/05/guia-vitimas-final.pdf.

24.Silva RM, Moraes-Filho IM, Valóta IAC, Saura APNS, Costa ALS, Sousa TV, et al. Nível de tolerância nas relações de amizade em profissionais de saúde durante a pandemia da COVID-19. REVISA. 2020; 9(Esp.1): 631-45. Doi: 10.36239/revisa.v9.nEsp1.p631a645

25. Henrique T. Covid-19 e a Internet (ou estou em isolamento social físico). Interfaces Cient Hum Soc. 2020;8(3):5-8. Doi: 10.17564/2316-3801.2020v8n3p5-8

Editora Científica Chefe: Cristiane Cardoso de Paula

Editora Científica: Tânia Solange Bosi de Souza Magnago

Fomento / Agradecimento: Não possui.

\section{Autor correspondente}

Rodrigo Marques da Silva

E-mail: Marques-sm@hotmail.com

Endreço: Rua Acre, Quadra 02, Lotes 17/18. Setor de Chácaras Anhanguera. Valparaíso de Goiás - GO

CEP: 72876-241 
17 | Moraes-Filho IM, Sousa TV, Carvalho-Filha FSS, Pereira MC, Vilanova JM, Silva RM

\section{Contribuições de Autoria}

\section{1 - Iel Marciano de Moraes Filho}

Colaborou com a concepção e projeto, análise e interpretação dos dados coletados, bem como redação do artigo e revisão crítica relevante do conteúdo intelectual.

\section{2 - Thais Vilela de Sousa}

Colaborou com a concepção e projeto, análise e interpretação dos dados coletados, bem como redação do artigo e revisão crítica relevante do conteúdo intelectual.

\section{3 - Francidalma Soares Sousa Carvalho Filha}

Colaborou na análise e interpretação dos achados, redação do artigo, assim como notável revisão crítica do conteúdo intelectual e aprovação final da versão final.

\section{4 - Mayara Cândida Pereira}

Colaborou na análise e interpretação dos achados, redação do artigo, assim como notável revisão crítica do conteúdo intelectual e aprovação final da versão final.

\section{5 - Jaiane de Melo Vilanova}

Colaborou na análise e interpretação dos achados, redação do artigo, assim como notável revisão crítica do conteúdo intelectual e aprovação final da versão final.

\section{6 - Rodrigo Marques da Silva}

Colaborou com a concepção e projeto, análise e interpretação dos dados coletados, bem como redação do artigo e revisão crítica relevante do conteúdo intelectual.

\section{Como citar este artigo}

Moraes-Filho IM, Sousa TV, Carvalho-Filha FSS, Pereira MC, Vilanova JM, Silva RM. Fatores sociodemográficos e emocionais associados a tolerância nas relações de amizade na pandemia pela COVID-19. Rev. Enferm. UFSM. 2021 [Acesso em: Anos Mês Dia]; vol.11 e2: 1-17. DOI:https://doi.org/10.5902/2179769253180 